\title{
Fifty years of Acta Neuropathologica: past, present, and future
}

\author{
Werner Paulus $\cdot$ Kurt A. Jellinger
}

Published online: 1 April 2011

(C) Springer-Verlag 2011

Acta Neuropathologica (ANP) was founded in 1961 by the renowned neurologist and neuropathologist Franz Seitelberger (1916-2007), then Director of the Neurological Institute (Obersteiner Institute) of the University of Vienna, in order to provide "a common forum accessible to all the neuropathology disciplines". In the preface to the first issue published in May 1961, Seitelberger emphasized the achievements of neuropathology based on the development of modern methods, including electron microscopy, histochemistry, biochemistry, cell culture, and neurophysiology. Seitelberger's idea was to create an international, interdisciplinary journal covering all available techniques and approaches in the wide field of neuroscience, resembling Heinrich Obersteiner's idea of the "International Brain Commission" as precursor of the International Brain Research Association (IBRO), also founded in 1961. ANP was suggested to continue "Veröffentlichungen des Neurologischen Instituts der Universität Wien" (Publications of the Neurological Institute, University of Vienna) or "Obersteiner Arbeiten" (Obersteiner Works) (1892-1923) at an international level. ANP became the official organ of the Research Groups for Neuropathology, Comparative Neuropathology and Neurooncology of the World Federation of Neurology. Major subjects during the initial decades were histopathology, (immuno)histochemistry, and surgical and experimental neuropathology, while

W. Paulus $(\bowtie)$

Institute of Neuropathology, University Hospital Münster, Domagkstr. 19, 48129 Munster, Germany

e-mail: werner.paulus@uni-muenster.de

K. A. Jellinger

Institute of Clinical Neurobiology, Kenyongasse 18,

1070 Vienna, Austria

e-mail: kurt.jellinger@univie.ac.at papers on molecular pathology have increased in later years. During the first years, four issues per year were published, rising in 1977 to six, and since 1988, to 12 issues per year. Between 1961 and 1983, eight supplements dealing with brain edema, neuroglia, pathology of axons and axonal flow, malignant lymphomas of the nervous system (papers of the first international symposium of malignant CNS lymphomas in Vienna), experimental and clinical neuropathology (papers of the First European Congress of Neuropathology in Vienna), and cerebrovascular transport mechanisms were published.

The first members of the editorial board of ANP (I. Bertrand, Paris, W. Blackwood, London, L. van Bogaert, Anvers, E. Frauchiger, Bern, W. Haymaker, Moffett Field, USA, W. Krücke, Frankfurt, F. Lüthy, Zurich, W. Scholz, Munich, all deceased) represented a "who's who" in neuropathology at that time. As Editor-in-Chief of ANP, Franz Seitelberger (1961-1987) was succeeded by Kurt A. Jellinger (1976-2004). They were often asked how to have managed the editorial activities for an international journal in addition to routine service and scientific work; Seitelberger also having the difficult tasks of being Dean of the Medical School and later Rector of the University of Vienna. However, enthusiasm overcame the daily work, and they became motivated by the progressing circulation and success of the journal. At that time, ANP and Current Contents were among the largest enimies in Jellinger's home, since he read all manuscripts and proofs during bathing and other activities, and $\mathrm{I}(\mathrm{KJ})$ have to apologize to my wife Elisabeth for this even after 50 years of happy marriage.

It is with pleasure to note that the hard work of the editors seems to have been worth the trouble. The impact factor has increased, and the current IF of 6.4 makes the journal number \#1 in neuropathology, \#3 among 71 
pathology journals, and \#6 among 167 clinical neurology journals of the ISI ranking. With respect to the immediacy index, reflecting the average number of citations of papers during the same year of publication and indicating how topical papers of a journal are, ANP runs even better (positions \#1, \#2 and \#3, respectively). Owing to the dedication of the editorial board, our external referees, and the publisher, turnaround times are now very short, with mean times from submission to first decision of 12 days, and from acceptance to online publication of 11 days in 2010 . The journal has very wide distribution, with more than 6,300 institutions worldwide having full-text online access. Color figures are free and there are no page charges. We do our best to provide authors and readers with the best service. Consequently, ANP is increasingly considered by neuropathologists and neuroscientists as the primary choice for publishing their best papers.

In an ever-faster changing world, it is impossible to predict where ANP will stand in the next 50 years. Clearly, the future position of ANP will depend on the status of the publishing business, the fate of the specialty of neuropathology, the role of science in general, and the major problems of society. We sincerely hope that the economic and ecologic conditions are beneficial, so that the pathogenesis, diagnosis, and management of neurological disease do not represent luxury issues (as is currently in some areas of the world) but remain in the center of the activities of a large number of clinical neuroscientists. The future of journal publishing is unpredictable as well. For example, it is conceivable that authors and scientific societies agree on large open-access publication platforms displacing most individual journals.

In the coming years, the content of ANP will largely be determined by the developments in neuropathology. On the one hand, neuropathology as a discipline studying the diagnosis and pathogenesis of neurological disorders is becoming increasingly important, because the population is growing older, we increasingly understand the molecular basis of brain disease leading to new therapy approaches, and because exciting developments in the ever-growing field of neuroscience bear implications for pathology. On the other hand, the impact of the neuropathologist's "core business", i.e., making a diagnosis based on histology, is expected to decrease, while the role of genetics, biomarker analysis, molecular imaging, and tissue-based molecular research will increase. The number of neurological lesions undergoing surgical resection and the amount of material derived from resections is not expected to increase, such as for vascular malformations, brain tumors, degenerative lesions of the spine, and others. The availability of autopsy materials is prone to political and philosophical issues which may change over time and may even result in a virtual lack of these tissues. Genetic analysis will further replace biopsy-based morphological analysis, such as in muscular disorders. Molecular markers might become more relevant to the management of patients with brain tumors than classical histopathology. Improvements of molecular imaging, e.g., with respect to classifying and grading of brain tumors, will put the role of histopathology into perspective. Finally, and most importantly, we believe that neuropathology will not be able to survive as an isolated diagnostic discipline without a strong focus on research. Currently, we encounter the perplexing situation that research in neuropathology is very active and successful in many nations, whereas the number of participants at national and international neuropathology meetings tends to decrease. In the same vein, many excellent papers from neuropathology departments are increasingly published in clinical neurology and neuroscience journals such as Brain, Annals of Neurology, Nature Neuroscience, and the Journal of Neuroscience, as well as in the top journals of science. This suggests that classical diagnostic neuropathology tends to diminish (albeit not yet being in danger of vanishing), whereas translational and interdisciplinary research in neuropathology is becoming ever more exciting and important, in part because of the unique expertise of neuropathologists with human disease and human tissue.

Neuropathology can no longer be defined primarily as the subject dealing with (morphological) diagnosis of nervous system disease. In order to survive, neuropathology as a diagnostic and research specialty needs to be supplemented with other areas such as genetics, molecular diagnostics, biomarker analysis, drug development, or molecular neuroimaging, to name just a few. This kind of reinvented neuropathology will play active and central roles in diagnosis and research. For Acta Neuropathologica, this means that we need to continue to broaden the scope and readership of the journal. We will aim for publishing the best papers on pathogenesis or diagnosis of neurological disease, and we encourage submissions bridging neuropathology with other neuroscience disciplines. In other words, we prefer papers by neuropathologists that are interesting to other neuroscientists, as well as papers by neuroscientists that are important to neuropathologists. Actually, this perfectly matches Franz Seitelberger's vision of neuropathology and its journal as outlined in his preface to the first issue 50 years ago: "Greater cooperation and interdisciplinary collaboration are needed if disease processes are to be placed in balanced perspective. There is no better way to obtain this perspective than for neuropathologists themselves to take a more active part in cultivating these fields." 